\title{
Scenarios for Ultrafast Gamma-Ray Variability in AGN
}

\author{
F. A. Aharonian ${ }^{1,2,3}$, M. V. Barkov ${ }^{4,5,6}$, and D. Khangulyan ${ }^{7}$ \\ ${ }^{1}$ Dublin Institute for Advanced Studies, 31 Fitzwilliam Place, Dublin 2, Ireland \\ ${ }^{2}$ Max-Planck-Institut für Kernphysik, Saupfercheckweg 1, D-69117 Heidelberg, Germany \\ ${ }^{3}$ National Research Nuclear University MEPhI, Kashirskoje Shosse, 31, 115409 Moscow, Russia \\ ${ }^{4}$ Deutsches Elektronen-Synchrotron (DESY), Platanenallee 6, D-15738 Zeuthen, Germany \\ 5 Astrophysical Big Bang Laboratory, RIKEN, 2-1 Hirosawa, Wako, Saitama 351-0198, Japan \\ ${ }^{6}$ Department of Physics and Astronomy, Purdue University, 525 Northwestern Avenue, West Lafayette, IN 47907-2036, USA \\ ${ }^{7}$ Department of Physics, Rikkyo University, Nishi-Ikebukuro 3-34-1, Toshima-ku, Tokyo 171-8501, Japan \\ Received 2017 March 8; revised 2017 April 24; accepted 2017 April 26; published 2017 May 24
}

\begin{abstract}
We analyze three scenarios to address the challenge of ultrafast gamma-ray variability reported from active galactic nuclei. We focus on the energy requirements imposed by these scenarios: (i) external cloud in the jet, (ii) relativistic blob propagating through the jet material, and (iii) production of high-energy gamma-rays in the magnetosphere gaps. We show that while the first two scenarios are not constrained by the flare luminosity, there is a robust upper limit on the luminosity of flares generated in the black hole magnetosphere. This limit depends weakly on the mass of the central black hole and is determined by the accretion disk magnetization, viewing angle, and the pair multiplicity. For the most favorable values of these parameters, the luminosity for 5-minute flares is limited by $2 \times 10^{43} \mathrm{erg} \mathrm{s}^{-1}$, which excludes a black hole magnetosphere origin of the flare detected from IC 310 . In the scopes of scenarios (i) and (ii), the jet power, which is required to explain the IC 310 flare, exceeds the jet power estimated based on the radio data. To resolve this discrepancy in the framework of scenario (ii), it is sufficient to assume that the relativistic blobs are not distributed isotropically in the jet reference frame. A realization of scenario (i) demands that the jet power during the flare exceeds by a factor $10^{2}$ the power of the radio jet relevant to a timescale of $10^{8}$ years.
\end{abstract}

Key words: galaxies: jets - galaxies: nuclei - gamma rays: galaxies - radiation mechanisms:nonthermal

\section{Introduction}

The hypothesis of supermassive black holes (SMBHs) as powerhouses of active galactic nuclei (AGNs) has been proposed (Salpeter 1964; Zel'dovich \& Novikov 1966; Lynden-Bell 1969) to explain the immense luminosities of AGN and quasars by the release of the gravitational energy through the process of gas accretion. The radiation power of the accreting plasma is limited by the Eddington luminosity, $L_{\mathrm{Edd}}=1.3 \times 10^{46} M_{8} \mathrm{erg} \mathrm{s}^{-1}$, where $M_{8}=M_{\mathrm{bh}} / 10^{8} M_{\odot}$ is the mass of the black hole in unities of $10^{8}$ solar masses.

The apparent luminosities of radiation of many AGN, $L_{\text {app }}=4 \pi D_{L}^{2} f$ ( $f$ is the detected energy flux and $D_{L}$ is the source luminosity distance) may exceed the Eddington luminosity of SMBHs by orders of magnitude. The "energy crisis" can be overcome if one assumes that the observed radiation is highly anisotropic, namely, that it is produced in a collimated outflow (jet) close to the line of sight (see, e.g., Begelman et al. 1984).

The concept of relativistically beamed emission offers not only an elegant scheme for the unification of various classes of AGN (see, e.g., Urry \& Padovani 1995), but also provides a natural interpretation of enormous fluxes of their nonthermal emission. Indeed, the assumption of production of radiation in a source relativistically moving toward the observer with a Doppler factor $\delta \gg 1$ allows physically reasonable intrinsic luminosities of AGN dubbed blazers, reducing them by orders of magnitude compared to the apparent luminosity, $L_{\text {app }}=\delta^{4} L_{\text {int }}$. This assumption also allows a larger (more "comfortable") size of the production region that is demanded by the observed variability of radiation: $l \leqslant c \Delta t_{\mathrm{var}} \delta$. These relations apply to all electromagnetic wavelengths, but they are crucial, first of all, for gamma-ray loud AGN, the apparent luminosities of which during strong flares, e.g., in 3C454.3 (Striani et al. 2010; Abdo et al. 2011) and 3C 279 (Hayashida et al. 2015), can achieve the level of $L_{\gamma} \sim 10^{49-50} \mathrm{erg} \mathrm{s}^{-1}$. Strong Doppler boosting is also needed to prevent severe internal gamma-ray absorption, especially at VHE energies (see, e.g., Celotti et al. 1998).

The gamma-ray emission of blazers is strongly variable, with fluxes that match the sensitivity of the Fermi Large Area Telescope (LAT) in the $\mathrm{MeV}-\mathrm{GeV}$ band well, and the current arrays of imaging atmospheric Cherenkov telescopes (IACT), H.E.S.S., MAGIC, Veritas, in the VHE band. During the strongest flares of BL Lac objects such as Mkn 421, Mkn 501, and PKS 2155-304, the energy fluxes of VHE gamma-rays often exceed $f_{\mathrm{VHE}}=10^{-10} \mathrm{erg} \mathrm{cm}^{-2} \mathrm{~s}^{-1}$. Such fluxes can be studied with IACT arrays with huge detection areas that are as large as $10^{5} \mathrm{~m}^{2}$ in an almost background-free regime, allowing variability studies on timescales of minutes. Although the fluxes of flaring powerful quasars at $\mathrm{MeV}-\mathrm{GeV}$ energies can be significantly larger, $f_{\mathrm{VHE}}=10^{-8} \mathrm{erg} \mathrm{cm}^{-2} \mathrm{~s}^{-1}$, because of the small detection area of space-borne instruments $\left(\simeq 1 \mathrm{~m}^{2}\right)$, the capability of the latter of probing the brevity of such strong AGN flares has until recently been limited by timescales of hours and days. However, after the release of the latest software tools by the Fermi LAT collaboration, which allow a significant increase in the gamma-ray photon statistics, the variability studies at $\mathrm{GeV}$ energies for exceptionally bright flares can be extended down to minute timescales. This potential has recently been demonstrated by the Fermi LAT collaboration for the giant 2015 June outburst of 3C 273 (Ackermann et al. 2016). 
It is straightforward to compare these timescales with the minimum time that characterizes a black hole system as an emitter, namely, the light-crossing time of the gravitational radius of the black hole:

$$
\tau_{0}=r_{\mathrm{g}} / c \approx 5 \times 10^{2} M_{8} \mathrm{~s} .
$$

Note that $r_{\mathrm{g}}=G M_{\mathrm{bh}} / c^{2}=1.5 \times 10^{13} M_{8} \mathrm{~cm}$ is the gravitational radius corresponding to the extreme Kerr black hole, i.e., twice smaller than the Schwarzschild radius.

Thus, for the mass range of black holes $M \geqslant 10^{8} M_{\odot}$, the current gamma-ray detectors have a potential to explore the physics of AGN that is close to the event horizon on timescales shorter than $\tau_{0}$. Such ultrafast gamma-ray flares ${ }^{8}$ have previously been detected from four AGN: PKS 2155 - 304 (Aharonian et al. 2007), Mkn 501 (Albert et al. 2007), and IC 310 (Aleksić et al. 2014) at TeV energies, and 3C 279 at $\mathrm{GeV}$ energies (Ackermann et al. 2016). In addition, a flare with a duration comparable to the $\mathrm{BH}$ horizon light-crossing time, $\sim 2 \tau_{0}$, was observed from a misaligned radio galaxy M87, in which the jet Doppler factor is expected to be small (Gebhardt \& Thomas 2009). For comparison, it is interesting to note that the characteristic timescales of even the shortest GRBs ( $1 \mathrm{~ms}$; Pozanenko \& Loznikov 2002; Golkhou et al. 2015), which are most likely associated with solar mass black holes, exceed $\tau_{0}$ by several orders of magnitude.

The detection of variable VHE gamma-ray emission from AGN on timescales significantly shorter than $\tau_{0}$ is an extraordinary result and requires a careful treatment and interpretation. The masses of SMBHs in distant AGN are typically derived from the empirical Faber-Jackson law (also known as the $M-\sigma$ relation, see Ferrarese \& Merritt 2000; Gebhardt et al. 2000). Although this statistical method is characterized by a small dispersion, scatter for individual objects may be significant, which consequently leads to uncertainties of $\tau_{0}$. On the other hand, it follows from Equation (2) that for the minute-scale flares reported from PKS 2155-304 and IC 310, the variability time can exceed $\tau_{0}$ only for masses of the BHs that are lower than $3 \times 10^{7} M_{\odot}$. For both objects, different methods of estimating $M_{\mathrm{bh}}$ give significantly higher values, and therefore $\tau<\tau_{0}$.

If the emission is produced in a relativistically moving source with a velocity $\beta_{\mathrm{em}}$, the variability timescale for the observer is shortened by the Doppler factor $\delta_{\mathrm{em}}=1 / \Gamma_{\mathrm{em}}\left(1-\beta_{\mathrm{em}} \cos \theta_{\mathrm{em}}\right)$; $\Gamma_{\mathrm{em}}=1 / \sqrt{\left(1-\beta_{\mathrm{em}}^{2}\right)}$ is the Lorentz factor and $\theta_{\mathrm{em}}$ is the angle between the source velocity and the line of sight. Thus if we wish to increase the proper size of the emitter $R^{\prime}$ (the source size in the comoving reference frame) to a physically reasonable value of $R^{\prime} \geqslant r_{\mathrm{g}}$, the Doppler factor should be large, $\delta_{\mathrm{em}}>10$. For example, in the case of PKS 2155-304, where the mass of SMBH is expected to be high, ${ }^{9} M_{8} \sim 10$, the VHE variability sets a lower limit on the value of the Doppler factor: $\delta_{\mathrm{em}} \geqslant 25$. However, there is another issue of conceptual importance that cannot be ignored. The problem is that if the perturbations originate in the central engine and then propagate in the jet, e.g., in the form of sequences of blobs ejected with different Lorentz factors (leading to internal shocks), the size of the emitter in the laboratory frame, $R=R^{\prime} / \Gamma_{\mathrm{j}}$, would not depend on the Doppler factor and it should exceed the gravitational radius: $R \geqslant r_{\mathrm{g}}$. Let

\footnotetext{
8 For a recent summary of ultrafast gamma-ray flares of AGN see Vovk \& Babić (2015).

9 To overcome this constraint, some models involve a BH binary system as the central engine in PKS 2155-304 (Rieger \& Volpe 2010).
}

us present the proper size of the production region as $R^{\prime}=\lambda \Gamma_{\mathrm{j}} r_{\mathrm{g}}$, where $\Gamma_{\mathrm{j}}$ is the jet bulk Lorentz factor, and $\lambda$ is a dimensionless parameter, which corresponds to the ratio of the production region size in the laboratory frame to the gravitational radius. The causality condition provides a limitation on the variability timescale

$$
t_{\mathrm{var}} \geqslant \tau_{0} \frac{\lambda \Gamma_{\mathrm{j}}}{\Gamma_{\mathrm{em}}} .
$$

The variability of $t_{\mathrm{var}}=0.04 \tau_{0}$ inferred from the VHE flares of PKS 2155-304 (Aharonian et al. 2007) requires $\Gamma_{\mathrm{em}} \simeq 25 \lambda \Gamma_{\mathrm{j}}$, i.e., the emitter should move relativistically in the frame of the jet, which in turn moves relativistically toward the observer. The jet-in-jet model suggested by Giannios et al. (2009) can be considered as a possible realization of this general scenario. Alternatively, if the source of the flare does not move relativistically relative to the jet $\left(\Gamma_{\mathrm{em}} \simeq \Gamma_{\mathrm{j}}\right)$, the size of the source in the laboratory frame should be much smaller than the black hole gravitational radius: $\lambda \simeq 0.04$.

If the emission site is located in the jet and formed by some perturbations propagating from the $\mathrm{BH}$, one should expect $\lambda>1$. Thus, the condition of $\lambda<1$ implies that the perturbations in the jet that result in a flare should have an external origin, i.e., are not directly linked to the central black hole. This scenario can be realized when a star or a gas cloud of radius $R_{*} \ll r_{\mathrm{g}}$ enters the jet from outside and initiates perturbations on scales smaller than the black hole gravitation radius $r_{\mathrm{g}}$ (Barkov et al. 2012a).

Finally, it has been suggested that the flares can be produced in the BH magnetosphere (Neronov \& Aharonian 2007; Levinson \& Rieger 2011; Rieger 2011). In this case, the production site does not move relativistically with respect to the observer, and Equation (2) is reduced to $t_{\mathrm{var}}>\tau_{0} \lambda$, where $\lambda=R / r_{\mathrm{g}}$. Thus, the flare originates in a compact region that occupies a small fraction of the black hole magnetosphere. An analogy for this possibility could be the emission of radioloud pulsars. It is believed that in these objects the radio pulses are produced in the polar cap region, which constitutes only a small part of the pulsar surface. Note that for the typical pulsar radius $R_{\mathrm{psr}}$ of $10 \mathrm{~km}, \tau_{0}=R_{\mathrm{psr}} / c \sim 30 \mu$ s is too small to be probed through the variability of the radio emission. We note here that although the production site of relativistic motion does not allow reducing the minimum variability time (see Equation (2)), the relativistic beaming effect allows significant relaxation of the energetics required to produce the flare. Thus, magnetospheric scenarios should have higher energy requirements than the jet scenarios.

In this paper we discuss in rather general terms three possible scenarios for the production of ultrafast ("subhorizon" scale) variability in AGNs:

(i) The source of the flare is a magnetospheric gap occupying a small volume in the proximity of the black hole close to the event horizon (Neronov \& Aharonian 2007; Levinson \& Rieger 2011).

(ii) The emitter moves relativistically in the jet reference frame. The most feasible energy source for this motion is magnetic field reconnection in a highly magnetized jet (Lyubarsky 2005; Giannios et al. 2009; Petropoulou et al. 2016).

(iii) Flares are initiated by penetration of external objects (stars or clouds) into the jet (Araudo et al. 2010; Barkov et al. 2012a). 
Apparently, any model designed to explain the ultrafast variability on timescales $t_{\mathrm{var}}<\tau_{0}$ should address some other key issues. In particular, the required overall energy budget should be feasible, the source should be optically thin for gamma-rays, and of course, the proposed radiation mechanism (s) should be able to explain the reported spectral features of gamma-ray emission.

\section{Addressing the "Subhorizon" Scale Variability}

\subsection{The Magnetospheric Model}

Magnetospheres of the central SMBHs in AGN can be sites of production of gamma-rays with spectra extending to VHE energies (see, e.g., Beskin et al. 1992; Levinson 2000; Neronov \& Aharonian 2007; Rieger \& Aharonian 2008; Levinson \& Rieger 2011). At low accretion rates, the injection of charges into the $\mathrm{BH}$ magnetosphere is not sufficient for a full screening of the electric field induced by the rotation of the compact object. The regions with unscreened electric field, referred to as gaps, are capable of effective acceleration of charged particles. Such a scenario may result in a variability of the source on "subhorizon" timescales since the size of the gap is much smaller than the gravitational radius. The attractiveness of this scenario is its applicability to the nonblazar-type AGN. On the other hand, because of both the low accretion rate and the lack of Doppler boosting, the gammaray luminosities of such objects are expected to be quite modest when compared to blazers. Therefore the detectability of the black hole magnetospheric radiation is most likely limited by a few nearby objects. In particular, the radio galaxy M87, as well as the compact radio source Sgr $\mathrm{A}^{*}$ in the center of our Galaxy, can be considered as suitable candidates for the realization of such a scenario (see, e.g., Levinson \& Rieger 2011).

The energy release in the entire magnetosphere is limited by the BZ luminosity. Below we follow a simplified treatment that allows us to estimate the energy release in a thin vacuum gap formed in the SMBH magnetosphere. The rotation of a magnetized neutron star or BH in vacuum induces an electric field, $\boldsymbol{E}_{0}$, in the surrounding space (Goldreich \& Julian 1969; Blandford \& Znajek 1977). If a charge enters this region, the electric field should accelerate it. In an astrophysical context the unscreened electric field is usually strong enough to boost the particle energy to the domain where the particle starts to interact with the background field and thus initiates an electron-positron pair cascade. The secondary particles move in the magnetosphere in a way that tends to screen the electric field (Sturrock 1971; Ruderman \& Sutherland 1975). Eventually, an electric-field-free configuration of the magnetosphere can be formed. However, one should note that there are differences between the structures of pulsar and BH magnetospheres, and consequently, the theoretical results obtained for pulsar magnetospheres cannot be directly applied to the $\mathrm{BH}$ magnetosphere. In particular, while in the case of the pulsar magnetosphere the source of the magnetic field is well defined, in the $\mathrm{BH}$ magnetosphere the magnetic field is generated by currents in the disk and magnetosphere. The configuration of the field is determined by the structure of the accretion flow. Thus, a change of the accretion flow can result in the formation of charge-starved regions (gaps) in the $\mathrm{BH}$ magnetosphere.
The charge density required for the screening is known as the Goldreich-Julian (GJ) density, $\rho_{\mathrm{GJ}}$ (Goldreich \& Julian 1969). However, the process of the pair creation is expected to be highly non-stationary (see, e.g., Levinson et al. 2005; Timokhin 2010 for theoretic and numerical considerations of the pair creation in vacuum gaps, respectively), thus even if an electric-field-free state of the magnetosphere is possible, it cannot be stable (Sturrock 1971). The gaps, i.e., regions in which the charge density is not sufficient for the electric field screening, may appear sporadically in the magnetosphere, for example, in the vicinity of the stagnation surface, i.e., at the boundary that separates acretion and ejection trends in the flow.

The vacuum electric field strength $\boldsymbol{E}_{0}$ determines the maximum electric field in the gap, thus the maximum acceleration rate of a particle with charge $e$ in the gap is $m c^{2} \dot{\gamma}<e c E_{0}$. The total power of particle acceleration can be expressed as

$$
\dot{\mathcal{E}}<\int_{\text {gap }} d V e\left(n_{\mathrm{e}}+n_{\mathrm{e}^{+}}\right) c E_{0}
$$

where $n_{\mathrm{e}}$ and $n_{\mathrm{e}^{+}}$are densities of electrons and positrons. If all the energy gained by the particles in the gap is emitted in gamma-rays, Equation (3) also corresponds to the upper limit of the gamma-ray luminosity. Note that electrons and positrons move in opposite directions in the gap, and only one of these species generates emission detectable by a distant observer.

For a thin spherical gap, $R<r<R+h$, the luminosity upper limit is

$$
L_{\gamma}<4 \pi R^{2} h e n_{\mathrm{e}} c E_{0}
$$

where the electrons are assumed to emit outward. The particle density can be expressed as a fraction of the Goldreich-Julian density: $e n_{\mathrm{e}}=\kappa \rho_{\mathrm{GJ}}$, where $\kappa$ is the multiplicity. The condition for the electric field screening, $e\left|n_{\mathrm{e}}-n_{\mathrm{e}^{+}}\right|=\rho_{\mathrm{GJ}}$, allows charge configurations with high multiplicity and still nonscreened electric field. To obtain a more detailed estimate of the generated pairs' influence on the electric field in the gap, it is necessary to consider the electromagnetic cascade in the gap.

The numerical simulations of Timokhin (2010) and Timokhin \& Arons (2013) demonstrate an important tendency. When the multiplicity becomes significant, $\kappa \sim 1$, the charges in the gap start to generate an electric field that is comparable to $E_{0}$, and the accelerating field vanishes. Thus, for effective charge acceleration, the following condition should be fulfilled: $\kappa \ll 1$. Thus, the total energy release in the gap of thickness, $h$, can be estimated as

$$
L_{\gamma}<4 \pi R^{2} h \kappa \rho_{\mathrm{GJ}} c E_{0} .
$$

The electrical field in the gap is estimated as

$$
E_{0} \approx B_{\mathrm{g}} \frac{R \Omega_{\mathrm{F}} \sin \theta}{c},
$$

where $B_{\mathrm{g}}$ is the magnetic field in the vacuum gap, $\Omega_{\mathrm{F}}$ is the angular velocity of the frame, $R$ is the radius, and $\theta$ is the polar angle. In fact, the actual electric field in the drop is smaller by a factor $h / R$ than the value given by Equation (6) (see, e.g., Blandford \& Znajek 1977; Levinson \& Rieger 2011). This factor accounts for the influence of the magnetospheric charges located outside the gap. Equation (6) does not account for this contribution. Since these charges, even if they remain outside 
the gap, tend to decrease the electrical field in the gap, Equation (6) provides a strict upper limit on the gap electric field strength.

The Goldreich-Julian density is also determined by the same parameters:

$$
\rho_{\mathrm{GJ}}=\Omega_{\mathrm{F}} B_{\mathrm{g}} \sin \theta /(2 \pi c) .
$$

For a Kerr $\mathrm{BH}$ with the maximum angular momentum, the angular velocity $\Omega_{\mathrm{F}}$ is estimated as

$$
\frac{\Omega_{\mathrm{F}}}{c} \simeq \frac{1}{4 r_{\mathrm{g}}}
$$

Substituting Equations (6)-(8) to Equation (5), one obtains

$$
L_{\gamma}<\frac{1}{8} \frac{B_{\mathrm{g}}^{2} R^{3}}{r_{\mathrm{g}}^{2}} \kappa h c \sin ^{2} \theta .
$$

The upper limit on the luminosity from a vacuum gap depends on the factor $R^{3} B_{\mathrm{g}}^{2}$ that is expected to decrease with $R$. For sake of simplicity below it is adopted that $R^{3} B_{\mathrm{g}}^{2} \simeq r_{\mathrm{g}}^{3} B_{\mathrm{bh}}^{2}$, where $B_{\mathrm{bh}}$ is the magnetic field at the $\mathrm{BH}$ horizon. Thus, one obtains

$$
L_{\gamma}<\frac{1}{8} B_{\mathrm{bh}}^{2} r_{\mathrm{g}} \kappa h c \sin ^{2} \theta .
$$

We should note that for $h \rightarrow r_{\mathrm{g}}$, the luminosity estimate provided by Equation (10) (after averaging over the polar angle $\theta$ ) exceeds the Blandford-Znajek (BZ) luminosity (Blandford \& Znajek 1977; Beskin 2010) by a factor of 2. This is imposed by several simplifications in our treatment. The most important contribution is caused by the usage of the electric field upper limit, Equation (6), as the accelerating field.

Thus, Equation (10) can be considered as a safe upper limit for the luminosity of magnetospheric flares. A similar estimate has been obtained by Rieger (2011) and Levinson \& Rieger (2011). However, the numerical expression in Levinson \& Rieger (2011) contains some uncertain geometrical factor ( $\eta$ in the notations of Levinson \& Rieger 2011). Equation (10) allows us to estimate its value: this geometrical factor should be small, $\sim 10^{-2}$ (see also Equation (52) in Rieger 2011).

Finally, Broderick \& Tchekhovskoy (2015) argued that for the full screening of the electric field in a thin gap, the charge density should exceed the Goldreich-Julian value by a factor ${ }^{10}$ $R / h$, which should lead to an enhancement of the gap radiation. To illustrate the physical reason for the existence of this factor, Broderick \& Tchekhovskoy (2015) computed the divergence of the electric field in the gap. However, as the gap electric field they adopted a field determined by an expression similar to Equation (6), i.e., a value that overestimates the true field by the factor $R / h$ (see, e.g., Blandford \& Znajek 1977; Levinson $\&$ Rieger 2011; Rieger 2011, for a more accurate introduction of the electric field in the gap). Thus, the factor suggested by Broderick \& Tchekhovskoy (2015) seems to be strongly overestimated.

Finally, the thickness of the gap, $h$, in Equation (10) is constrained by the variability timescale, $h \sim t_{\mathrm{var}} c$. To produce the emission variable on a 5-minute timescale, $t_{\mathrm{var}}=5 t_{\mathrm{var}, 5}$ minutes, the gap thickness, $h=10^{13} t_{\mathrm{var}, 5} \mathrm{~cm}$, should be smaller than the gravitational radius of the $\mathrm{SMBH}$ with a mass $M_{8}>1$. Thus, the

\footnotetext{
${ }^{10}$ Note that Broderick \& Tchekhovskoy (2015) used a different notation for the gap thickness, $\Delta$.
}

estimated gamma-ray luminosity cannot exceed the following value:

$$
L_{\gamma}<5 \times 10^{43} \kappa B_{4}^{2} M_{8} t_{\mathrm{var}, 5} \sin ^{2} \theta \mathrm{erg} \mathrm{s}^{-1}
$$

where $B_{\text {bh }}=10^{4} B_{4} \mathrm{G}$.

Equation (11) contains two parameters that are determined by properties of the advection flow in the close vicinity of the $\mathrm{BH}$ : pair multiplicity, $\kappa$, and the magnetic field strength, $B$. Importantly, these parameters are essentially defined by the same property of the flow, more specifically, by the accretion rate. The magnetic field at the $\mathrm{BH}$ horizon needs to be supported by the accretion flow. Therefore the field strength is directly determined by the accretion rate. The accretion rate also defines the intensity of photon fields in the magnetosphere, and consequently, the density of electron-positron pairs produced through gamma-gamma interaction (see, e.g., Levinson \& Rieger 2011). If the multiplicity parameter, $\kappa$, approaches unity, the gap electric field vanishes (see, e.g., Timokhin \& Arons 2013). This sets an upper limit on the accretion rate, and consequently on the magnetic field strength.

In previous studies (Levinson \& Rieger 2011; Aleksić et al. 2014) the maximum accretion rate compatible with the existence of a vacuum gap in the magnetosphere was estimated as

$$
\dot{m}<3 \times 10^{-4} M_{8}^{-1 / 7}
$$

where $\dot{m}$ is the accretion rate in Eddington units:

$$
\dot{M}_{\mathrm{edd}}=\frac{4 \pi m_{\mathrm{p}} G M_{\mathrm{bh}}}{\eta c \sigma_{\mathrm{t}}} .
$$

Here $m_{\mathrm{p}}, \sigma_{\mathrm{t}}$, and $\eta$ are the proton mass, the Thompson crosssection, and the accretion efficiency factor, respectively.

To derive the estimate provided by Equation (12), Levinson $\&$ Rieger (2011) adopted a value of $\eta=0.1$ and estimated the magnetic field strength at the $\mathrm{BH}$ horizon as

$$
B_{\text {bh }}=1.3 \times 10^{5}\left(\dot{m} / M_{8}\right)^{1 / 2} G
$$

where we rescaled the numerical coefficient to the normalization used throughout our paper. For this magnetic field strength, Equation (11) yields

$$
L_{\gamma}<3 \times 10^{42} \kappa M_{8}^{-1 / 7} t_{\mathrm{var}, 5} \sin ^{2} \theta \mathrm{erg} \mathrm{s}^{-1} .
$$

In some cases, e.g., for IC 310, the energy requirements are rather close to the obtained upper limit, therefore we consider a somewhat more accurate treatment of the case of a magnetosphere around a Kerr BH below.

The strength of the magnetic field at the $\mathrm{BH}$ horizon can be obtained by extrapolating the field at the last marginally stable orbit. Let us define the magnetic field in the disk as

$$
B_{\mathrm{d}}=\sqrt{8 \pi \beta_{\mathrm{m}} p_{\mathrm{g}}},
$$

where $\beta_{\mathrm{m}}$ and $p_{\mathrm{g}}$ are the disk magnetization and gas pressure in the accretion disk that confines the magnetic field at the horizon. The gas pressure can be estimated using the solution 
for a radiatively inefficient accretion flow (see Narayan \& Yi 1994) ${ }^{11}$ as

$$
p_{\mathrm{g}}=\frac{\sqrt{10} \dot{M} \sqrt{G M_{\mathrm{bh}}}}{12 \pi \alpha_{\mathrm{ss}} R^{5 / 2}},
$$

where $\alpha_{\mathrm{ss}}$ is the nondimensional viscosity of the disk (Shakura $\&$ Sunyaev 1973). Equation (16) for $R \rightarrow r_{\mathrm{g}}$ provides an estimate for the magnetic field at the $\mathrm{BH}$ horizon:

$$
B_{\mathrm{bh}}=1.5 \frac{\beta_{\mathrm{m}}^{1 / 2}(\dot{M} c)^{1 / 2}}{\left(\alpha_{\mathrm{ss}}\right)^{1 / 2} r_{\mathrm{g}}} .
$$

The magnetic field strength provided by Equation (18) together with Equation (10) yields

$$
L_{\gamma}<\frac{\sqrt{10}}{12} \frac{\beta_{\mathrm{m}} \kappa\left(h / r_{\mathrm{g}}\right) \sin ^{2} \theta \dot{M} c^{2}}{\alpha_{\mathrm{ss}}} .
$$

The multiplicity parameter, $\kappa$, at the Kerr radius is determined as (see Appendix A for details)

$$
\kappa \equiv \frac{n_{ \pm}}{n_{\mathrm{GJ}}} \approx 6 \times 10^{6} \frac{\dot{m}^{7 / 2} M_{8}^{1 / 2}}{\left(\eta \alpha_{\mathrm{ss}}\right)^{7 / 2} \beta_{\mathrm{m}}^{1 / 2}} .
$$

The condition $\kappa<1$ determines an upper limit on the accretion rate:

$$
\dot{m}<10^{-2} \frac{\eta \alpha_{\mathrm{ss}} \beta_{\mathrm{m}}^{1 / 7}}{M_{\mathrm{bh}}^{1 / 7}} .
$$

Equations (21) and (13) substituted into Equations (18) and (19) give an upper limit for the magnetic field that is consistent with the existence of vacuum gaps:

$$
B_{\text {bh }}<7 \times 10^{3}\left(\frac{\beta_{\mathrm{m}}}{M_{8}}\right)^{4 / 7} \mathrm{G},
$$

and consequently, the maximum luminosity of particles accelerated in the gap does not depend on $\alpha_{\mathrm{ss}}$ and $\eta$ :

$$
L_{\gamma}<2 \times 10^{43} \beta_{\mathrm{m}}^{8 / 7} \kappa t_{\mathrm{var}, 5} M_{8}^{-1 / 7} \sin ^{2} \theta \operatorname{erg~s}^{-1} .
$$

This estimate is obtained for the thick-disk accretion (in the ADAF-like regime). The limit on the accretion rate given by Equation (12) is consistent with the realization of this accretion regime. For higher accretion rates, $\dot{m} \geqslant 0.1$, the accretion flow is expected to converge to the thin-disk solution (BisnovatyiKogan \& Blinnikov 1977; Abramowicz et al. 1988). In this regime, the temperature of the disk is expected to be significantly below $1 \mathrm{MeV}$, thus the pair creation by photons supplied by the accretion disk should cease. This effectively mitigates the constraints imposed by the accretion rate. However, the change of the accretion regime also significantly weakens the strength of the magnetic field at the $\mathrm{BH}$ horizon (Bisnovatyi-Kogan \& Lovelace 2007), and consequently decreases the available power for acceleration in the gap.

To derive Equation (23), we assumed that the gap thickness is determined by the variability timescale; this corresponds to the energetically most feasible configuration. In a more realistic treatment, one should also should take into account the

\footnotetext{
11 A more accurate treatment of the accretion flow reveals a correction by less than 30\% (see Narayan \& Yi 1995a) compared to the height-averaged treatment in Narayan \& Yi (1994).
}

interaction of the particles into account that are accelerated in the gap with the background radiation field. For high and ultrahigh energies of electrons, $E>1 \mathrm{TeV}$, the characteristic time of the inverse Compton scattering appears to be shorter than the minute-scale typical for the short $\mathrm{TeV}$ flares (see Appendix A). For the hot target photon field, as expected from a thick accretion disk, the pair-production process should also be very efficient, $\lambda_{\gamma \gamma} \leqslant \lambda_{\text {IC. }}$. Thus, computation of the TeV emission requires a detailed modeling of the electromagnetic cascade (see, e.g., Broderick \& Tchekhovskoy 2015; Hirotani et al. 2016). Furthermore, the production and evacuation of the cascade-generated pairs may follow a cyclic pattern and the inductive electric field may become comparable to the vacuum field (Levinson et al. 2005). A detailed consideration of this complex dynamics is beyond the scope of this paper, but we note that the characteristic length of such a cascade-moderated gap should be small, $\sim \sqrt{\lambda_{\text {IC }} \lambda_{\gamma \gamma}}$, resulting in a reduction of the available power (see also Beskin et al. 1992).

Equation (23) determines the maximum luminosity of vacuum gaps that can collapse quicker than $t_{\mathrm{var}}$. It has been assumed for its derivation that the magnetic field is determined by an accretion regime that in turn determines the intensity of the photon field in the magnetosphere. In the case of steady accretion, this seems to be a very feasible approximation. However, this may look less certain in the case of a rapidly changing accretion rate, since the processes that govern the variation of the accretion rate and escape of the magnetic field from the $\mathrm{BH}$ horizon may have different characteristic timescales. Therefore we provide some estimates for these two timescales below.

The dominant contribution to the photon field comes from plasma located at distances $r \sim 2 r_{\mathrm{g}}$, and the characteristic viscous accretion time (density decay time in the flow) is

$$
t_{\rho, \text { decay }} \simeq \frac{2 r_{\mathrm{g}}}{c \alpha_{\mathrm{ss}}} \simeq 10^{4} \alpha_{\mathrm{ss},-1}^{-1} M_{8} \mathrm{~s} .
$$

When the accretion fades, the decay of the magnetic field is determined by the magnetic field reconnection rate (Komissarov 2004):

$$
t_{\mathrm{B}, \mathrm{decay}} \simeq \frac{\pi r_{\mathrm{g}}}{0.3 c} \sim 10^{4} M_{8} \mathrm{~s} .
$$

Since these two timescales are essentially identical, it is natural to expect that the field strength and the disk density will decay simultaneously. Thus, Equation (23) should also be valid for the time-dependent accretion regime.

\subsection{Relativistically Moving Blobs}

The properties of radiation generated in jets may be significantly affected if some jet material moves relativistically with respect to the jet local comoving frame. For example, the magnetic field reconnection may be accompanied by the formation of slow shocks (see, e.g., Lyubarsky 2005) that in the magnetically dominated plasma produce relativistic flows (Komissarov 2003). If such a process is realized in AGN jets, it can lead to gamma-ray flares in blazar-type AGN with variability timescales significantly shorter than $r_{\mathrm{g}} / c$ (Giannios et al. 2009). Another implication of this scenario is related to short gamma-ray flares detected from misaligned radio galaxies (Giannios et al. 2010). Indeed, the conservation of momentum requires that for each plasmoid directed within the jet-opening 
cone, there should exist a counterpart that is directed outside the jet-beaming cone. While the radiation of the plasmoid directed along the jet appears as a short flare, the emission associated with its counterpart outflow can be detected as a bright flare by an off-axis observer. The latter process may have a direct implication on the interpretation of flares from nearby misaligned radio galaxies, e.g., M87 (Giannios et al. 2010).

If a process, operating in a region of the jet with comoving volume $V^{\prime}$, results in the ejection of plasmoids, some fraction, $\xi$, of the energy contained in the volume is transferred to the outflow. The conservation of energy can be written as

$$
\xi V^{\prime} \epsilon_{\mathrm{j}}^{\prime}=S_{\mathrm{co}} \Gamma_{\mathrm{co}}^{2} v_{\mathrm{co}} \Delta t^{\prime}\left(4 / 3 \tilde{\epsilon}_{\mathrm{e}}\right) .
$$

Here $\epsilon_{\mathrm{j}}^{\prime}$ and $\Delta t^{\prime}$ are the energy density of the jet plasma and duration of the ejection, as seen in the jet comoving reference frame. $\Gamma_{\mathrm{co}}=1 / \sqrt{1-\left(v_{\mathrm{co}} / c\right)^{2}}, S_{\mathrm{co}}$, and $\tilde{\epsilon}$ are the plasmoid Lorentz factor, the outflow cross-section, and the internal energy, respectively. The outflow cross-section can be estimated as $S_{\mathrm{co}} \simeq S /\left(2 \Gamma_{\mathrm{co}}^{2}\right)$, where $S \simeq V^{\prime} /\left(\Delta t^{\prime} v_{\mathrm{co}}\right)$ is the surface of the volume $V^{\prime}$. Thus, one obtains an estimate for the energy density of

$$
\epsilon_{\mathrm{j}}^{\prime} \simeq \frac{2}{3 \xi} \tilde{\epsilon}_{\mathrm{e}}
$$

For simplicity, in what follows we take $\xi \epsilon_{\mathrm{j}}^{\prime} \simeq \tilde{\epsilon}_{\mathrm{e}}$. The efficiency of the energy transfer, $\xi$, depends on a specific realization of the scenario. For example, it seems that for the reconnection of the magnetic field, the efficiency might be high $\xi \sim 1$, as follows from an analytic treatment by Lyubarsky (2005) and the results of numerical simulations by Sironi et al. (2016). ${ }^{12}$ We note, however, that in the presence of a guiding field, the magnetization of the ejected plasmoids should be high (Lyubarsky 2005).

On the other hand, the energy density in the plasmoid can be estimated through the emission variability time and the luminosity level (see Equation (9) Giannios et al. 2009):

$$
\tilde{\epsilon}_{\mathrm{e}}=\frac{E_{\mathrm{em}}}{\Gamma_{\mathrm{em}} \tilde{l}_{\mathrm{em}}^{3}}
$$

where the variability timescale determines the size of the production region: $\tilde{l}_{\mathrm{em}}=c \Delta t \Gamma_{\mathrm{em}}$, and the flux level defines the energy content in the plasmoid: $E_{\mathrm{em}}=L_{\gamma} \Delta t /\left(4 f \Gamma_{\mathrm{em}}^{2}\right)$ (here $f<1$ defines the fraction of the plasmoid energy transferred to the flare emission; this factor is dropped in what follows, and its contribution is accounted for in the value of the factor $\xi$ ). Thus, one obtains

$$
\tilde{\epsilon}_{\mathrm{e}}=\frac{L_{\gamma}}{4 \Gamma_{\mathrm{em}}^{6} c^{3} \Delta t^{2}} .
$$

On the other hand, the energy density in the jet is

$$
\epsilon^{\prime}{ }_{\mathrm{j}}=\frac{L_{\mathrm{j}}}{\Delta \Omega r^{2} c \Gamma_{\mathrm{j}}^{2}}
$$

\footnotetext{
12 From Figure 2 of Sironi et al. (2016) it follows that $n_{\text {lab }} \simeq \sigma n_{0}$ and $\langle\gamma\rangle_{\text {lab }} n_{\text {lab }} \simeq \sigma^{2} n_{0}$, thus the internal energy in the plasmoid is $\tilde{\epsilon} \simeq \tilde{n}\langle\tilde{\gamma}\rangle \simeq \sigma n_{0} m c^{2}$.
}

where $\Delta \Omega \simeq \pi / \Gamma_{\mathrm{j}}^{2}$ is the jet propagation solid angle. The comparison of these equations allows us to estimate the required true luminosity of the jet as

$$
L_{\mathrm{j}}=\frac{L_{\gamma}}{\Gamma_{\mathrm{em}}^{6}} \frac{\pi r^{2}}{4 \xi c^{2} \Delta t^{2}} .
$$

The above equation is consistent with Equation (10) from Giannios et al. (2009). Note, however, a difference in the notations: throughout this paper, $L_{\mathrm{j}}$ is the true jet luminosity, while in Giannios et al. (2009) $L_{\mathrm{j}}$ corresponds to the isotropic luminosity.

If the viewing angle is small, the mini-jet Lorentz factor can be expressed as $\Gamma_{\mathrm{em}}=2 \Gamma_{\mathrm{j}} \Gamma_{\mathrm{co}} /\left(1+\alpha^{2}\right)$, where $\alpha=\theta \Gamma_{\mathrm{j}}$ is the viewing angle expressed through the jet-opening angle (see Appendix B). Thus, the above equation can be simplified as

$$
L_{\mathrm{j}}=\frac{L_{\gamma}}{\Gamma_{\mathrm{co}}^{6} \Gamma_{\mathrm{j}}^{6}} \frac{\left(1+\alpha^{2}\right)^{6}}{256} \frac{\pi r^{2}}{\xi c^{2} \Delta t^{2}}
$$

or

$$
L_{\mathrm{j}}=1.4 \times 10^{-5} L_{\gamma}\left(\frac{1+\alpha^{2}}{4}\right)^{6} \Gamma_{\mathrm{co}, 1}^{-6} \Gamma_{\mathrm{j}, 1}^{-6} \xi_{-1}^{-1} r_{2}^{2} M_{8}^{2} t_{\mathrm{var}, 5}^{-2}
$$

Here it was assumed that the flare originates at a distance $r_{2}=100 r_{\mathrm{g}}$ from the central BH with mass $M_{\mathrm{bh}}=10^{8} M_{\odot} M_{8}$.

The above estimate describes the jet luminosity requirement to generate a single short flare of duration $t_{\mathrm{var}}$. Observations in $\mathrm{HE}$ and VHE regimes show that AGNs often demonstrate a rather long period of activity (as compared to the duration of a single peak): $T \gg t_{\mathrm{var}}$. If the mini-jets are isotropically distributed in the jet comoving frame, the probability for an observer to be in the mini-jet beaming cone depends weakly on the observer viewing angle ${ }^{13}$, and this probability can be estimated as $P \simeq\left(2 \Gamma_{\text {co }}\right)^{-2}$ (Giannios et al. 2010). If the minijet formation is triggered by some spontaneous process, then the comoving size of the region responsible for the flare is $l_{0}^{\prime}=\delta_{\mathrm{j}} T c$, and the energy contained in this region is $E^{\prime}=S l_{0}^{\prime} e_{\mathrm{j}}^{\prime}$ (here $S$ is the jet cross-section). The energy of a single mini-jet in the comoving frame is

$$
E_{\mathrm{mj}}^{\prime}=\frac{L_{\gamma} t_{\mathrm{var}} \Gamma_{\mathrm{co}}}{4 \xi \Gamma_{\mathrm{em}}^{3}} .
$$

The total number of mini-jets during a flaring episode can be estimated as $N \approx \Phi T / P t_{\text {var }}$, where $\Phi$ is the so-called filling factor. The total dissipated energy for the flare should be smaller than the energy that is contained in the dissipation region:

$$
\frac{E_{\mathrm{mj}}^{\prime} \Phi T}{P t_{\mathrm{var}}}<L_{\mathrm{j}} T \frac{\delta_{\mathrm{j}}}{\Gamma_{\mathrm{j}}^{2}} .
$$

This implies a requirement for the jet luminosity

$$
L_{\mathrm{j}}>0.1 \Phi \zeta^{2} \delta_{\mathrm{j}, 1}^{-2} L_{\gamma} \xi_{-1}^{-1},
$$

here $\zeta=\Gamma_{\mathrm{j}} / \delta_{\mathrm{j}}$, or

$$
L_{\mathrm{j}}>0.006 \Phi\left(1+\alpha^{2}\right)^{4} \Gamma_{\mathrm{j}, 1}^{-2} L_{\gamma} \xi_{-1}^{-1}
$$

\footnotetext{
${ }^{13}$ If $\Gamma_{\mathrm{co}}>\Gamma_{\mathrm{j}}$, this statement is correct for observers located in $\theta_{\text {view }}<\pi / 2$, otherwise for $\tan \theta_{\text {view }}<v_{\mathrm{co}} / \sqrt{1 / \Gamma_{\mathrm{co}}^{2}-1 / \Gamma_{\mathrm{j}}^{2}}$.
} 
where the small viewing angle limit was used for the ratio of Lorentz and beaming factors: $\zeta=\Gamma_{\mathrm{j}} / \delta_{\mathrm{j}} \simeq\left(1+\alpha^{2}\right) / 2$ (see Appendix B). The requirement imposed by Equation (36) significantly exceeds the limit related to the shortest variability time, Equation (33).

The derived lower limit for the jet luminosity contains the parameter $\xi$, which accounts for the conversion efficiency from jet material to the outflow, and from outflow to the radiation. While the latter can be high, $\sim 1$, if a good target for nonthermal particles exists, the value of the former efficiency depends on the process behind the outflow formation. For example, it was argued that if the outflow is formed by Petschek-type relativistic reconnection (Lyubarsky 2005), the energy transfer is expected ${ }^{14}$ to be high, $\sim 1$. However, the efficiency of the transfer can be significantly suppressed if the guiding field is present in the reconnection domain (Lyubarsky 2005; Barkov \& Komissarov 2016).

On the other hand, this requirement can be somewhat relaxed if the velocity direction of the plasmoids is not random, e.g., is controlled by the large-scale magnetic field (Giannios et al. 2009), or is triggered by some perturbation propagating from the base of the jet. In the former case the mini-jet detection probability, $P$, may be higher, and in the latter case, the comoving distance between the mini-jets may be larger than $l_{0}^{\prime}$. Let us assume that the flare trigger propagates with a Lorentz factor $\Gamma_{\text {tr }}^{\prime}$ in the jet comoving frame, then the comoving region size is larger by a factor of $\Gamma_{t r}^{\prime}$.

\subsection{Cloud-in-jet Model}

In the framework of the cloud-in-jet scenario, we deal with the nonthermal emission generated at the interaction of a jet with some external obstacle, e.g., a BLR cloud or a star (see, e.g., Blandford \& Königl 1979; Bednarek \& Protheroe 1997; Araudo et al. 2010; Barkov et al. 2010, 2012b). Debris of the obstacle matter, produced at such an interaction, can be caught by the jet flow. This debris should form dense blobs or clouds in the jet, and the emission generated during their acceleration may be detected as a flare (Barkov et al. 2012a; Khangulyan et al. 2013). If this interpretation is correct, each peak of the light curve can be associated with emission produced at the acceleration of some individual blob. The peak profile and its duration are determined by the condition of how quickly this blob can be involved in the jet motion, i.e., by the dimension and mass of the blob. Light blobs with a mass satisfying the condition

$$
M_{\mathrm{c}} c^{2}<\frac{P_{\mathrm{j}} \pi R_{\mathrm{c}}^{2} r_{0}}{4 \Gamma_{\mathrm{j}}^{3}}
$$

(here $P_{\mathrm{j}}$ and $R_{\mathrm{c}}$ are the jet ram pressure and the cloud radius, respectively) are accelerated on length scales smaller than the distance to the $\mathrm{SMBH}, r_{0}$ (for a consideration of heavy blobs that can be accelerated over a distance comparable to $r_{0}$, see Khangulyan et al. 2013).

\footnotetext{
${ }^{14}$ This follows from Equation (6) in (Giannios et al. 2009), i.e., $\tilde{\epsilon}_{\mathrm{e}}=\sigma \rho_{\mathrm{j}}^{\prime} c^{2} \simeq \epsilon_{\mathrm{j}}^{\prime}$ for $\sigma \gg 1$.
}

If this condition is fulfilled, the variability timescale can be estimated as (Barkov et al. 2012a; Khangulyan et al. 2013)

$$
t_{\mathrm{var}} \simeq \frac{4 c M_{\mathrm{c}} \Gamma_{\mathrm{j}}^{2}}{P_{\mathrm{j}} \pi R_{\mathrm{c}}^{2} \delta_{\mathrm{j}}}
$$

This estimate ignores perturbations in the jet that are generated by the obstacle-jet interaction, which is probably very complex, and their influence can be explored only by numerical simulations, which are beyond the scope of this paper (see, e.g., de la Cita et al. 2016).

In the case of a cloud with a mass that satisfies Equation (38), the variability can be very short: $t_{\mathrm{var}}<r_{0} /\left(c \Gamma_{\mathrm{j}} \delta_{\mathrm{j}}\right)$, but obviously it cannot be shorter than the light-crossing time of the blob: $t_{\mathrm{var}}>R_{\mathrm{c}}^{\prime} /\left(c \delta_{\mathrm{j}}\right)$. Equation (39) implies that there is a critical minimum mass of the blob below of which the dynamics of the blobs is very quick and the variability is limited by the blob light-crossing limit.

On the other hand, the mass of the blob determines the energy transferred by the jet to the blob during its acceleration, and consequently, the apparent energy emitted in the corresponding peak of the light curve (Khangulyan et al. 2013):

$$
E_{\gamma} \simeq \xi M_{\mathrm{c}} c^{2} \delta_{\mathrm{j}}^{3}
$$

where the factor $\xi$ accounts for the fraction of energy transferred to the gamma-ray emitting particles and some dynamical factor $(\sim 0.3$, which is related to the correction function $F_{\mathrm{e}}$ defined in Barkov et al. 2012a; Khangulyan et al. 2013). Thus, if the cloud dynamics determines the variability, then the luminosity of the emission appears to be independent of the mass of the cloud:

$$
L_{\gamma} \simeq c P_{0} \pi R_{\mathrm{c}}^{2} \frac{\xi \delta_{\mathrm{j}}^{4}}{4 \Gamma_{\mathrm{j}}^{2}} .
$$

Since $L_{\mathrm{j}}>c P_{0} \pi R_{\mathrm{c}}^{2}$, the above equation allows us to obtain a lower limit on the jet luminosity required for the operation of the star-jet interaction scenario:

$$
L_{\mathrm{j}}>0.4 \zeta^{2} \delta_{\mathrm{j}, 1}^{-2} L_{\gamma} \xi_{-1}^{-1}
$$

or

$$
L_{\mathrm{j}}>0.025\left(1+\alpha^{2}\right)^{4} \Gamma_{\mathrm{j}, 1}^{-2} L_{\gamma} \xi_{-1}^{-1},
$$

which is a factor of $4 / \Phi$ larger than the estimate for the jet-injet scenario (see Equation (36)).

Equations (39) and (40) contain four parameters $P_{0}, \Gamma_{\mathrm{j}}$ (we treat $\delta_{\mathrm{j}}$ as a related parameter), $M_{\mathrm{c}}$, and $R_{\mathrm{c}}$, and therefore formally allow a solution even if two of these parameters are fixed. For example, for given properties of the jet (i.e., for specific values of the parameters $\Gamma_{\mathrm{j}}$ and $P_{0}$ ) and the parameters characterizing the flare (the total energy and the variability), the characteristics of the cloud can be determined as

$$
M_{\mathrm{c}} c^{2}=\frac{E_{\gamma}}{\xi \delta_{\mathrm{j}}^{3}}, \quad \pi R_{\mathrm{c}}^{2}=\frac{4 \zeta^{2}}{\delta_{\mathrm{j}}^{2}} \frac{E_{\gamma}}{\xi t_{\mathrm{var}} c P_{0}} .
$$

However, the determined parameters of the cloud may not necessary be physical, and their feasibility should be examined by dynamical estimates.

The first dynamical limitation is related to the ability of a cloud to penetrate the jet and become involved in the jet 
Table 1

Comparison of Models for Different Sources

\begin{tabular}{|c|c|c|c|c|c|}
\hline$\overline{\text { Source }}$ & $\overline{\text { IC } 310^{\mathrm{a}}}$ & ${\mathrm{M} 87^{\mathrm{b}}}^{\mathrm{b}}$ & 30 C $454.3^{\mathrm{c}}$ & $=3 \mathrm{C} 279^{\mathrm{d}}$ & 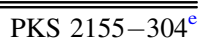 \\
\hline$M_{\mathrm{BH}, 8}$ & 3 & 60 & 10 & 5 & 10 \\
\hline$t_{5}$ & 1 & 175 & 54 & 8.4 & 0.6 \\
\hline$\tau_{0}$ & 0.2 & 2 & 3 & 1 & 0.04 \\
\hline$\Phi$ & 0.1 & 0.3 & 0.7 & 0.3 & 0.7 \\
\hline$\Gamma_{\mathrm{j}}$ & 10 & 10 & 20 & 20 & 20 \\
\hline$L_{\gamma} / L_{\gamma, \mathrm{ms}}$ & 10 & $5 \times 10^{-4}$ & $3 \times 10^{5}$ & $5 \times 10^{4}$ & $10^{4}$ \\
\hline$L_{\mathrm{j}, \mathrm{jj}}$ & $10^{44}$ & $10^{42}$ & $2 \times 10^{47}$ & $3 \times 10^{45}$ & $10^{44}$ \\
\hline$L_{\mathrm{j}, \mathrm{cj}}$ & $3 \times 10^{45}$ & $2 \times 10^{43}$ & $10^{48}$ & $4 \times 10^{46}$ & $6 \times 10^{44}$ \\
\hline
\end{tabular}

Notes. $M_{\mathrm{BH}, 8}=M_{\mathrm{BH}} / 10^{8} M_{\odot}$ is the SMBH mass, $t_{5}=t / 300 \mathrm{~s}$ is the variability time, $\tau_{0}=t c / r_{\mathrm{g}}$ is the nondimensional variability time in units of gravitation radius light-crossing time, $L_{\gamma}$ is the maximum luminosity in gamma-rays, $\Gamma_{\mathrm{j}}$ is the jet Lorentz factor, $\Gamma_{\mathrm{co}}$ is the Lorentz factor of the mini-jet, $\alpha=\theta / \Gamma_{\mathrm{j}}$ is the normalized viewing angle, $L_{\gamma, \mathrm{ms}}$ is the upper limit of the gamma-ray luminosity for a magnetospheric model, $L_{\mathrm{j}, \mathrm{jj}}$ is the minimal jet power for the jet-in-jet model, $L_{\mathrm{j}, \mathrm{cj}}$ is the minimum jet power for the cloud-in-jet model.

${ }^{a}$ Aleksić et al. (2014).

b Gebhardt \& Thomas (2009), Wang \& Zhou (2009), Abramowski et al. (2012).

${ }^{\mathrm{c}}$ Striani et al. (2010), Abdo et al. (2011).

${ }^{\mathrm{d}}$ Hayashida et al. (2015).

e Aharonian et al. (2007).

motion. According to the estimates given by Barkov et al. (2012a) and Khangulyan et al. (2013), for the typical jet parameters these constraints do not impose any strong limitations. The heaviest blobs that can be accelerated by a jet with luminosity $10^{43} \mathrm{erg} \mathrm{s}^{-1}$ can result in flares with a total energy release of $10^{54} \mathrm{erg}$.

If the cloud is light enough to be caught by the jet, then one should consider two main processes: the cloud expansion, and its acceleration. At the initial stage, the cloud cross-section is not sufficiently large to provide its acceleration to relativistic velocities. On the other hand, the intense jet-cloud interaction at this stage leads to a rapid heating and expansion of the cloud. The cloud size-doubling time can be estimated as

$$
t_{\exp } \approx A\left(\frac{M_{\mathrm{c}}}{\gamma_{\mathrm{g}} R_{\mathrm{c}} P_{\mathrm{j}}}\right)^{1 / 2}
$$

where $\gamma_{\mathrm{g}}=4 / 3$ is the adiabatic index and $A$ is a constant of about a few (Gregori et al. 2000; Nakamura et al. 2006; Pittard et al. 2010; Bosch-Ramon et al. 2012). When the size of the cloud becomes large enough for acceleration to relativistic energies, the intensity of the jet-cloud interaction fades away, and the cloud expansion proceeds in the linear regime. Since the timescale for acceleration to relativistic velocity is

$$
t_{\mathrm{ac}} \simeq \frac{M_{\mathrm{c}} c^{2}}{\pi R_{\mathrm{c}}^{2} c P_{\mathrm{j}}},
$$

the size of the cloud relevant for the flare generation can be obtained by balancing Equations (45) and (46):

$$
R_{\mathrm{c}}=A_{\exp }\left(\frac{M_{\mathrm{c}} c^{2}}{P_{\mathrm{j}}} \frac{\gamma_{\mathrm{g}}}{\pi^{2} A^{2}}\right)^{1 / 3} \text {. }
$$

Here the constant $A_{\text {exp }}$ accounts for the cloud expansion in the linear regime.
The dynamical limitation given by Equation (47) together with Equation (44) allows determination of the jet ram pressure:

$$
P_{\mathrm{j}}=\frac{\pi A^{4}}{\xi \gamma_{\mathrm{g}}^{2} A_{\exp }^{6}}(2 \zeta)^{6} \frac{E_{\gamma}}{t_{\mathrm{var}}^{3} c^{3}} .
$$

The actual value of the coefficient in the above equation, in particular the value of $A_{\exp }$, can be revealed only through numerical simulations given the complexity of the jet-cloud interaction. However, if one assumes that the expansion proceeds very efficiently, i.e., the cloud size achieves a value close to the light-crossing limit, $R_{\mathrm{c}} \simeq \delta_{\mathrm{j}} t_{\mathrm{var}} c$, then the expression for the jet ram pressure becomes

$$
P_{\mathrm{j}}=\left(\frac{2 \zeta}{\delta_{\mathrm{j}}^{2}}\right)^{2} \frac{E_{\gamma}}{\pi \xi t_{\mathrm{var}}^{3} c^{3}} .
$$

Since each flaring episode should correspond to specific jet parameters, ${ }^{15}$ the above equation implies that the energy emitted in an individual peak of a flare should be proportional to the cube of its duration: $E_{\gamma} \propto t_{\mathrm{var}}^{3}$ (or $L_{\gamma} \propto t_{\mathrm{var}}^{2}$ ). Obviously, the study of individual peaks in a statistically meaningful way requires a detailed light curve that can be obtained with future observations, in particular with CTA (see, e.g., Romoli et al. 2017).

\subsection{Energetic Constraints for Detected Exceptional Flares}

So far, several super-fast gamma-ray flares have been detected in VHE or HE regimes from different types of AGNs. The peculiarity of the signal is related both to the duration of

\footnotetext{
${ }^{15}$ We note, however, that across a magnetically driven jet one may expect strong gradients of the jet ram pressure (see, e.g., Beskin \& Nokhrina 2006; Komissarov et al. 2007, 2009).
} 
the flare and to the released energy. Below we consider several cases that are summarized in Table 1.

\subsubsection{PKS $2155-304$}

The July 2006 flare of PKS 2155-304 is characterized by a very short variability of $180 \mathrm{~s}$ and an intrinsic VHE gamma-ray luminosity at the level of $10^{47} \mathrm{erg} \mathrm{s}^{-1}$ (Aharonian et al. 2007).

The mass of the central $\mathrm{BH}$ is estimated to be $M_{\mathrm{BH}, 8} \simeq 10$ (Aharonian et al. 2007, and reference therein). Together with the short variability time, this constrains the luminosity of (potential) gamma-ray flares produced by magnetosphere gaps at the level of $L_{\gamma, \mathrm{ms}}<10^{43} \mathrm{erg} \mathrm{s}^{-1}$ and thus excludes any magnetospheric origin of these flares.

PKS 2155-304 is a typical representative of high-energy peaked BL Lacs. It is expected that the jet is aligned along the line of sight: $\alpha \approx 0$. For a typical value of the jet Lorentz factor, $\Gamma_{j}=20$, the jet power required for the realization of the jet-in-jet scenario is

$$
L_{\mathrm{j}, \mathrm{jj}}>10^{44} \Phi_{-0.2} \Gamma_{\mathrm{j}, 1.3}^{-2} \xi_{-1}^{-1}
$$

It follows from the comparison of Equations (37) and (43) that the lower limit of the jet power in the cloud-in-jet scenario is higher by a factor $\sim 4 / \Phi$, i.e.,

$$
L_{\mathrm{j}, \mathrm{cj}}>6 \times 10^{44} \Gamma_{\mathrm{j}, 1.3}^{-2} \xi_{-1}^{-1} \mathrm{erg} \mathrm{s}^{-1} .
$$

We note here that constraints imposed by the radiation mechanism enhance the required jet power by a factor of $\sim 10$ for the external inverse Compton scenario and by $\sim 10^{2}$ for proton synchrotron emission (see Barkov et al. 2012a), which exceeds the Eddington luminosity.

$$
\text { 2.4.2. IC } 310
$$

In 2012 November, the MAGIC collaboration detected a bright flare from IC 310 (Aleksić et al. 2014). The flare consisted of two sharp peaks with a typical duration of $\sim 5$ minutes. The measured spectra were hard, with a photon index $\lesssim 2$, extending up to $\sim 10 \mathrm{TeV}$. The energy released during this event has been estimated to be at a level of $2 \times 10^{44} \mathrm{erg} \mathrm{s}^{-1}$.

The mass of the $\mathrm{BH}$ powering activity of IC 310 has been estimated to be $M_{\mathrm{IC} 310}=\left(3_{-2}^{+4}\right) \times 10^{8} M_{\odot}$ (Aleksić et al. 2014), i.e., the measured variability timescale is as short as $20 \%$ of $\tau_{0}$.

According to the estimate provided by Equation (23), the luminosity of flares generated in the $\mathrm{BH}$ magnetosphere depends weakly on the mass of the $\mathrm{BH}$ and is determined by the disk magnetization, the viewing angle, and the pair multiplicity. ${ }^{16}$ Since all these parameters are smaller than unity, from Equation (23) we have

$$
L_{\gamma, \mathrm{ms}}<2 \times 10^{43} \mathrm{erg} \mathrm{s}^{-1} .
$$

This upper limit is an order of magnitude below the required value (Aleksić et al. 2014). Thus, we conclude that the ultrafast flare detected from this source cannot have a magnetospheric origin.

Assuming that mini-jets are distributed isotropically in the jet frame and that the detection of two pulses is not a statistical

\footnotetext{
${ }^{16}$ Equation (23) does not account for relativistic effects, which should be small unless the gap is formed close to the horizon. However, if the vacuum gap is close to the horizon, then the gravitational redshift should make the constraints imposed by the variability time more reliable.
}

fluctuation, one can estimate the true jet luminosity using Equation (37). For the relevant flare parameters (i.e., $t_{\mathrm{var}}=4.8 \mathrm{~min}, L_{\gamma}=2 \times 10^{44} \mathrm{erg} \mathrm{s}^{-1}$ ) and $M_{8}=3$

$$
L_{\mathrm{j}, \mathrm{jj}}>10^{44} \Phi_{-1}\left(\frac{1+\alpha^{2}}{5}\right)^{4} \Gamma_{\mathrm{j}, 1}^{-2} \xi_{-1}^{-1}
$$

If the mini-jets are not distributed isotropically, the requirement on the jet power can be a few orders of magnitude weaker; see Equation (33).

The cloud-in-jet scenario requires a higher jet luminosity; from Equation (43) it follows that

$$
L_{\mathrm{j}, \mathrm{cj}}>3 \times 10^{45} \Gamma_{\mathrm{j}, 1}^{-2}\left(\frac{1+\alpha^{2}}{5}\right)^{4} \xi_{-1}^{-1} \mathrm{erg} \mathrm{s}^{-1}
$$

$$
\text { 2.4.3. } M 87
$$

In 2010, a bright flare has been recorded during a multiinstrument campaign in the VHE energy band (Abramowski et al. 2012). The variability time during the VHE transient was about 0.6 day and the flux level achieved $10^{42} \mathrm{erg} \mathrm{s}^{-1}$. This source is characterized by a large jet-viewing angle of $\theta_{\mathrm{j}} \approx 15^{\circ}$ and a Lorentz factor of about $\Gamma_{\mathrm{j}} \approx 7$ (Wang \& Zhou 2009), and the SMBH mass is $\sim 6 \times 10^{9} M_{\odot}$ (Gebhardt \& Thomas 2009).

Given the heavy central $\mathrm{BH}$ and the relatively long duration of the VHE flare, which allows high values of the gap size, the energy constraint in the magnetosphere scenario is quite modest:

$$
L_{\gamma, \mathrm{ms}}<2 \times 10^{45} \mathrm{erg} \mathrm{s}^{-1} \text {. }
$$

M87 might be an interesting candidate for a detection of magnetosphere flares.

For the flare parameters (i.e., $t_{\mathrm{var}}=0.6$ days, $L_{\gamma}=10^{42} \mathrm{erg} \mathrm{s}^{-1}$ ) and $M_{8}=60$, Equation (37) constrains the required jet true luminosity at the level

$$
L_{\mathrm{j}, \mathrm{jj}}>10^{42} \Phi_{-0.5}\left(\frac{1+\alpha^{2}}{5}\right)^{4} \Gamma_{\mathrm{j}, 1}^{-2} \xi_{-1}^{-1} .
$$

On the other hand, the mulitwavelength properties of the gamma-ray flares detected from M87 seem to be quite diverse, with no detected robust counterparts at other wavelengths. Thus, if the VHE emission is produced by a single mini-jet, then a much weaker constraint, provided by Equation (33), is applied. In this case, the variability detected with Cherenkov telescopes should correspond to the mini-jet variability, thus the mini-jet comoving size should be

$$
\tilde{l}_{\mathrm{em}}=\Delta t c \Gamma_{\mathrm{em}}=\frac{2 \Delta t c \Gamma_{\mathrm{j}} \Gamma_{\mathrm{co}}}{1+\alpha^{2}} \sim 10^{17} \mathrm{~cm},
$$

which is about the jet cross-section at a parsec distance from the central BH. We should also note that the typical spectra emitted by plasmoids are dominated by synchrotron radiation, which seems to be inconsistent with the multiwavelength observations of M87. Moreover, the peculiar light curve that has been detected with H.E.S.S. has not yet been explained in the framework of the jet-in-jet scenario.

Formally, for the parameters of the flare detected from M87, the minimum jet luminosity required by the cloud-in-jet 
scenario is

$$
L_{\mathrm{j}, \mathrm{cj}}>2 \times 10^{43} \Gamma_{\mathrm{j}, 1}^{-2}\left(\frac{1+\alpha^{2}}{5}\right)^{4} \xi_{-1}^{-1} \mathrm{erg} \mathrm{s}^{-1} .
$$

However, it has been argued that the light curve and the VHE spectrum is best explained if the $\mathrm{TeV}$ is produced through $\mathrm{p}-\mathrm{p}$ interactions induced by the jet collision with a dense cloud. In this case, the required jet power is about $L_{\mathrm{j}} \approx 5 \times 10^{44} \mathrm{erg} \mathrm{s}^{-1}$ (Barkov et al. 2012b).

\subsection{4. $3 C 454.3$ and $3 C 279$}

In 2010 November, an exceptionally bright flare was detected from 3C 454.3 by AGILE and Fermi LAT (Striani et al. 2010; Abdo et al. 2011). The minumum detected variability time and gamma-ray luminosity were $4.5 \mathrm{hr}$ and $2 \times 10^{50} \mathrm{erg} \mathrm{s}^{-1}$, respectively.

Several similarly bright flares were detected form 3C 279 in the period from 2013 December to 2014 April (Hayashida et al. 2015). The GeV gamma-ray luminosity reached a level of $6 \times 10^{48} \mathrm{erg} \mathrm{s}^{-1}$, and the flux varied on a timescale of $0.7 \mathrm{hr}$.

The magnetosphere gap luminosity is limited by $10^{45} \mathrm{erg} \mathrm{s}^{-1}$ and $2 \times 10^{44} \mathrm{erg} \mathrm{s}^{-1}$ for $3 \mathrm{C} 454.3$ and $3 \mathrm{C} 279$, respectively. Therefore the magnetospheric origin of these flares is excluded for both sources.

3C 279 and 3C 454.3 are distant quasars, thus it is safe to fix $\alpha=0$ for both cases. By adopting a standard jet Lorentz factor, $\Gamma_{j}=20$, one can obtain the jet luminosity required for the realization of the jet-in-jet scenario:

$$
L_{\mathrm{j}, \mathrm{jj}}>10^{47} \Phi_{-0.2} \Gamma_{\mathrm{j}, 1.3}^{-2} \xi_{-1}^{-1}
$$

for $3 \mathrm{C} 454.3$, and

$$
L_{\mathrm{j}, \mathrm{jj}}>3 \times 10^{45} \Phi_{-0.5} \Gamma_{\mathrm{j}, 1.3}^{-2} \xi_{-1}^{-1}
$$

for 3C 279. Both these estimates appears to be below the corresponding Eddington luminosity limits of $1.3 \times 10^{47} \mathrm{erg} \mathrm{s}^{-1}$ and $6 \times 10^{46} \mathrm{erg} \mathrm{s}^{-1}$ for $M_{\mathrm{BH}, 8}=10$ and $M_{\mathrm{BH}, 8}=5$ in $3 \mathrm{C} 454.3$ and $3 \mathrm{C} 279$, respectively.

The star-in-jet scenario requires higher jet luminosities, which seem to exceed the Eddington limit for 3C 454.3. Namely, one obtains

$$
L_{\mathrm{j}, \mathrm{cj}}>10^{48} \Gamma_{\mathrm{j}, 1.3}^{-2} \xi_{-1}^{-1} \mathrm{erg} \mathrm{s}^{-1} .
$$

This value agrees with the estimate $L_{\mathrm{j}, \mathrm{cj}} \approx 10^{49} \mathrm{erg} \mathrm{s}^{-1}$ that is obtained within a more accurate model that also provides an interpretation for the so-called plateau phase and the spectrum (Khangulyan et al. 2013). Such a high luminosity of the jet, $L_{\mathrm{j}} \approx 0.1 L_{\gamma}$, has also been obtained in the framework of the one-zone external Compton model of Bonnoli et al. (2011).

For 3C 279, the lower limit on the jet luminosity is

$$
L_{\mathrm{j}, \mathrm{cj}}>4 \times 10^{46} \Gamma_{\mathrm{j}, 1.3}^{-2} \xi_{-1}^{-1} \operatorname{erg~s}^{-1},
$$

which is close to the Eddington limit. A similar estimate was obtained by Hayashida et al. (2015).

\section{Discussion and Conclusions}

\subsection{Gamma-ray Flare Detected from IC 310}

IC 310 is a radio galaxy with redshift $z \simeq 0.0189$ (Falco et al. 1999). Radio observations revealed an extended jet with a viewing angle of $\theta_{\mathrm{j}}<30^{\circ}$ (see, e.g., Kadler et al. 2012). Arguments based on the absence of a contra-jet and assuming that the true length of the jet is smaller than $1 \mathrm{Mpc}$ allowed to further constrain the viewing angle $10^{\circ}<\theta_{\mathrm{j}}<20^{\circ}$ (for details see Aleksić et al. 2014). Finally, observations of superluminal motion allowed us to constraint the Doppler factor to $\delta_{\mathrm{j}} \sim 5$. The radio luminosity has been estimated to be at the level of $L_{\mathrm{IC} 310 \text {, radio }} \simeq 10^{41} \mathrm{erg} \mathrm{s}^{-1} \quad$ (Sijbring \& de Bruyn 1998). This implies a minimum energy in relativistic electrons of $5.61 \times 10^{57} \mathrm{erg}$, thus the power required for the supply of emitting electrons yields $L_{\mathrm{IC} 310, \mathrm{e}} \simeq 2 \times 10^{42} \mathrm{erg} \mathrm{s}^{-1}$, and the total jet luminosity can be estimated as $L_{\mathrm{IC} 310} \simeq 10^{43} \mathrm{erg} \mathrm{s}^{-1}$ (see Aleksić et al. 2014). We note that these estimates represent values for the minimum required energetics averaged over $10^{8}$ years.

In 2012 November, MAGIC detected a bright flare from IC 310 (Aleksić et al. 2014). The flare consisted of two sharp peaks with a typical duration of $\sim 5$ minutes. The measured spectrum was hard, with a photon index of $\lesssim 2$, extending up to $\sim 10 \mathrm{TeV}$. The energy released during that event has been estimated to be at a level of $2 \times 10^{44} \mathrm{erg} \mathrm{s}^{-1}$.

The mass of the $\mathrm{BH}$ powering activity of IC 310 has been determined to be $M_{\mathrm{IC} 310}=\left(3_{-2}^{+4}\right) \times 10^{8} M_{\odot}$ (Aleksić et al. 2014), i.e., the measured variability timescale is as short as $20 \%$ of $\tau_{0}$; therefore one can expect a realization of some unconventional mechanism for VHE emission production. Aleksić et al. (2014) have considered possible scenarios (see Section 2) for the flare production and found that jet-in-jet and star-in-jet interaction models face certain difficulties. Based on this, one concluded that the magnetosphere origin remains the only possible option, and no further verification of that scenario has been provided.

Hirotani \& Pu (2016) have performed simulations of the gamma-ray spectrum produced in a stationary gap for different accretion rates and concluded that the emission generated in the vacuum gap could closely reproduce the spectral properties of the $\mathrm{TeV}$ emission detected during the flare.The strength of the magnetic field has been fixed at a level of $10^{4} \mathrm{G}$. Hirotani \& $\mathrm{Pu}$ (2016) have emphasized that the generation of such a strong magnetic field requires an accretion rate exceeding the values compatible with the existence of vacuum gaps by a factor of 100. In addition, we note that if the thickness of the gap is determined by the variability time, $h \simeq t_{\mathrm{var}} c$, this scenario requires an even higher efficiency (by a factor of $\sim 20$, since the best-fit parameters for the magnetospheric scenario require $\dot{m} \sim 5 \times 10^{-6}$ and consequently $h_{\text {gap }} \sim 5 r_{\mathrm{g}}\left(\sim 25 t_{\mathrm{var}} c\right)$, see Figures 8 and 17 of Hirotani \& Pu 2016).

Since one does not expect any significant focusing or enhancement of the emission produced in the magnetosphere, the measured energy should correspond to the real energetics of the processes responsible for the emission generation. Thus, the feasibility of generating such a powerful flare in the vacuum gap is closely related to the general efficiency of processes taking place in $\mathrm{BH}$ magnetosphere. Currently, the BZ mechanism (Ruffini \& Wilson 1975; Lovelace 1976; Blandford \& Znajek 1977) represents the most prominent energy extraction mechanism that can operate in the $\mathrm{BH}$ magnetosphere. The efficiency of this mechanism is determined by the strength of the magnetic field that is accumulated at the $\mathrm{BH}$ horizon, which in turn is determined by the accretion rate. Hirotani \& Pu (2016) argued that the efficiency of the BZ mechanism can be very high, up to a level of $900 \%$, as compared to the accretion rate $\dot{M} c^{2}$. This assumption is based 
on 2D simulations presented by McKinney et al. (2012). However, 3D simulations for a similar setup presented in the same paper reveal a significantly lower efficiency, $\sim 300 \%$. We note that Equation (19) with $\alpha_{\mathrm{ss}}=0.1$ corresponds to an efficiency of $250 \%$, which is very close to the results of the 3D simulations by McKinney et al. (2012).

According to the estimate provided by Equation (23), the possible luminosity of flares generated in $\mathrm{BH}$ magnetosphere depends very weakly on the mass of the $\mathrm{BH}$ and is determined by disk magnetization, viewing angle, and pair multiplicity. ${ }^{17}$ Since all these parameters are smaller than one, the numerical coefficient in Equation (23) can be taken as a strict upper limit for the flare luminosity for the given variability time. This upper limit appears to be approximately an order of magnitude below the value measured with MAGIC (Aleksić et al. 2014). Thus, we conclude that it seems very unfeasible that these processes are indeed behind the bright flaring activity recorded from IC 310 (which is also consistent with modeling presented by Hirotani et al. 2016).

Our simplified analysis does not allow us to robustly rule out two other scenarios for the flare production in IC 310. If one adopts the minimum averaged jet luminosity as a reasonable constraint for the present jet luminosity (as done in Aleksić et al. 2014), then both scenarios formally do not allow reproducing the observed properties. However, if one assumes that there is some anisotropy in the mini-jet distribution, then jet-in-jet model provides an energetically feasible scenario. We note that the spectral energy distributions currently obtained for the jet-in-jet models feature a dominant excess in the UV band (Petropoulou et al. 2016), which is not consistent with the observations from IC 310 . This might be either a fundamental constraint or just a systematic underestimation of the inverse Compton contribution that is due to the small scale of the simulations. Thus, it seems that more detailed large-scale simulations are required to verify the applicability of the jet-injet scenario for IC 310. On the other hand, if the present-day jet luminosity is significantly higher than the averaged value, the star-in-jet scenario may also meet the energetic requirements.

\subsection{Comparison of Scenarios for Ultrafast Variability}

In this paper, we considered three scenarios for the production of ultrafast AGN flares with variability times shorter than the Kerr radius light-crossing time: gamma-ray emission of gaps in the SMBH magnetosphere (Neronov \& Aharonian 2007; Levinson \& Rieger 2011), the jet-in-jet realization (Giannios et al. 2009), and the emission caused by penetration of external dense clouds (Barkov et al. 2012a).

The production of gamma-rays in the $\mathrm{BH}$ magnetosphere has several unique properties. In particular, this scenario can be invoked to explain emission from off-axis AGNs and orphan gamma-ray flares. On the other hand, the luminosity of the magnetospheric gap has a robust upper limit that depends weakly on the SMBH mass. Moreover, the magnetospheric emission is not enhanced by the Doppler-boosting effect, and this seems to be crucial for explaining short flares from distant AGN. On the other hand, some nearby SMBHs (Levinson \& Rieger 2011), e.g., the Sagittarius A star or M87, might be very

\footnotetext{
17 Equation (23) does not account for relativistic effects, which should be small unless the gap is formed close to the horizon. If the vacuum gap is close to the horizon, then gravitational redshift should even strengthen the constraints imposed by the variability time.
}

promising candidates to produce gamma-ray flares (see, however, Li et al. 2009; Levinson \& Rieger 2011; Cui et al. 2012 for the discussion of gamma-gamma attenuation in magnetosphere).

In general terms, there can be little doubt that the nonthermal radiation of powerful $\mathrm{AGN}$ is related, in one way or another, to relativistic jets. The ultrafast gamma-ray flares might be linked to the formation of relativistically moving features (plasmoids or mini-jets) inside the major outflow, the jet originating from the central black hole. Depending on the orientation of the mini-jets to the jet axis, the radiation of the mini-jet can be focused within the jet cone or outside. This scenario has been suggested to interpret the variable emission from AGN (Giannios et al. 2009, 2010). It has been shown that under certain conditions, magnetic field reconnection can result in the formation of relativistic outflows (Lyubarsky 2005; Sironi et al. 2016). We note, however, that the formation of a relativistic outflow is not an indispensable feature of reconnection. Thus, ejection of relativisitcally moving plasmoids may require a specific configuration of the magnetic field. Independently, to form outflows with large Lorentz factors, $\Gamma_{\text {co }} \geqslant 10$, an initial configuration with high magnetization, $\sigma \simeq \Gamma_{\text {co }}^{2} \geqslant 100$, is required. Such a high magnetization of the jet at the flare production site requires an even higher initial jet magnetization, $\sigma_{\text {init }} \gg 10^{3}$. Jets with such a high magnetization should have an extremely low mass load, which seems to be inconsistent with the properties of AGN jets at large distances (see, however, Komissarov 1994; Stern \& Poutanen 2006; Araudo et al. 2013 and references therein).

Finally, the SED of the emission produced by plasmoids formed at reconnection contains a dominating synchrotron component that peaks in the UV energy band (Petropoulou et al. 2016). This feature is not consistent with the SEDs obtained from AGNs during the ultrafast flares. The presence of a guiding magnetic field can significantly enhance the magnetization of plasmoids, resulting in a further enhancement of the synchrotron component and perhaps in the extension of the synchrotron component to the gamma-ray band. The examination of this scenario requires detailed modeling, since the guiding filed also impacts the Lorentz factor of plasmoids.

The jet-in-jet scenario quantitatively implies a modest requirement for the jet intrinsic luminosity, however; it can be even further relaxed if one assumes that the mini-jets are not distributed isotropically in the major jet comoving frame. Such an anisotropy can be realized, for example, by focusing the outflow along the direction of the reconnecting magnetic field.

An important issue to realize the jet-in-jet scenario is the triggering mechanism for the reconnection. If the jet is launched by the BZ mechanism, it is expected to be magnetically dominated at the initial stage, thus reconnection is a thermodynamicaly favored process. However, the reasoning based on equipartition arguments, without any particular energy transfer mechanism, can hardly be valid. The timescale of such thermodynamic processes may be enormous; thus they might be irrelevant for astrophysical jets. In recent years, significant progress has been achieved in PIC simulations for the reconnection in magnetic field configurations with alternating polarities. Such configurations should naturally appear in the pulsar outflows close to the current sheet. However, it is less obvious how such regions would form in AGN jets. Several scenarios can be considered. The first is a change in the magnetic field polarity in the jet caused by a change 
in magnetic field polarity in the accretion disk, and consequently, in the BH magnetosphere (McKinney \& Uzdensky 2012; Barkov \& Baushev 2011). However, such a change takes a long time, of about $\sim 10^{3} \mathrm{r}_{\mathrm{g}} / c$, and it is hard to expect ultrafast variability caused by such a configuration of the magnetic field. An arrangement with alternating magnetic field polarities can also be a result of a growth of MHD instabilities in the jet (see, e.g., Barniol Duran et al. 2016). However, an intense instability growth leads to a flow disruption on a scale of several dynamical lengths (Porth \& Komissarov 2015). So it is hard to obtain an intensive reconnection event close to the base of a jet that extends a significant distance beyond the reconnection region. Finally, the reconnection can be caused by a sudden compression and mixing of a small part of the jet, which, for example, can be due to an external obstacles in the jet. In such a case, a short but intensive local reconnection episode may occur without disrupting the entire flow. This specific case represents an interesting synergy of two models: the formation of a relativistic mini-jet by reconnection of the magnetic field triggered by a star in the jet. The feasibility of this scenario needs to be tested with detailed numerical simulations, however.

The star-in-jet scenario, the third possibility considered in the paper, requires significantly higher jet luminosity than the jet-injet scenario. In many cases, the jet luminosity, needed to realize the star-in-jet scenario, exceeds the Eddington limit. It was also shown that some details of the $\mathrm{GeV}$ light curve obtained from 3C 454.3 with Fermi LAT, e.g., the plateau phase, can be readily interpreted in the framework of the star-injet scenario (Khangulyan et al. 2013). It is also important to note that the emission produced by the interaction of a cloud with the AGN jet should be characterized by a universal relation between the luminosity and the duration of individual peaks of the flare: $L^{1 / 2} \propto \Delta t$. To verify this relation observationally, a high photon statistics is required, which may possibly be achieved with future observations with CTA.

The authors are grateful to the anonymous referee for the insightful comments and helpful suggestions. We would like to thank Andrey Timokhin and Frank Rieger for productive discussions. The authors appreciate the support by the Russian Science Foundation under grant 16-12-10443. D.K. acknowledges financial support by a grant-in-aid for Scientific Research (KAKENHI, No. 24105007-1) from the Ministry of Education, Culture, Sports, Science and Technology of Japan (MEXT). M.B. acknowledges partial support by the JSPS (Japan Society for the Promotion of Science): No. 2503786, 25610056, 26287056, 26800159. M.B. also acknowledges MEXT (Ministry of Education, Culture, Sports, Science and Technology): No. 26105521.

\section{Appendix A \\ Calculation of the Pair Multiplicity from a Radiatively Inefficient Accretion Flow}

Levinson \& Rieger (2011) have estimated the density of electron-positron pairs produced by photon-photon annihilation in a radiatively inefficient accretion flow (RIAF, Narayan \& Yi 1995b). For the sake of consistency, a similar consideration is presented below, but for a Kerr $\mathrm{BH}$ and explicitly accounting for the nondimensional viscosity parameter $\alpha_{\mathrm{ss}}$ and radiation efficiency of an accretion flow $\eta$. Identically to Levinson \& Rieger (2011), we relie on the solution obtained by
Narayan \& Yi (1995b), assuming that the advection parameter is small: $1-f \ll 1$ (with notations of Narayan \& Yi 1995b). In particular, the radial velocity of the accretion flow is taken as

$$
v_{\mathrm{r}}=\frac{3 \alpha_{\mathrm{ss}}}{5}\left(\frac{G M_{\mathrm{bh}}}{r}\right)^{1 / 2} \text {. }
$$

The ion density $n_{\mathrm{i}}$ can be estimated as

$$
n_{\mathrm{i}}(r)=\frac{\dot{M}}{4 \pi r H m_{\mathrm{p}} v_{\mathrm{r}}}=\frac{5 \sqrt{10}}{6} \frac{\dot{m}}{\eta \alpha_{\mathrm{ss}}} \frac{\left(G M_{\mathrm{bh}}\right)^{1 / 2}}{c \sigma_{\mathrm{T}} r^{3 / 2}},
$$

given the revealed height of the accretion disk, $H \simeq c_{\mathrm{s}} / \Omega_{\mathrm{k}}$ (for details and notations see Narayan \& Yi 1994).

The total cooling rate of the ion-electron plasma can be estimated (Narayan \& Yi 1995b; Levinson \& Rieger 2011) as

$$
q_{f f}=q_{\mathrm{ee}}+q_{\mathrm{ei}} \approx 10^{-21} n_{\mathrm{e}}^{2} \theta_{\mathrm{e}} \mathrm{erg} \mathrm{s}^{-1} \mathrm{~cm}^{-3}
$$

for relativistic electron temperature $\theta_{\mathrm{e}}=k T_{\mathrm{e}} / m_{\mathrm{e}} c^{2} \gtrsim 1$. Since the density of the ions exceeds the GJ density, the pair production does not provide any sensible contribution to the disk electron density in configurations allowing the existence of vacuum gaps, thus in what follows we assume the number densities of electrons and ions to be equal, $n_{\mathrm{i}}=n_{\mathrm{e}}$.

For such a hot electron plasma the emission appears in the $\mathrm{MeV}$ energy band, and the luminosity of the inner part of the accretion flow is (Levinson \& Rieger 2011)

$$
L_{f f} \approx \int_{r_{\mathrm{g}}}^{2 r_{\mathrm{g}}} 2 \pi r^{2} q_{f f} d r .
$$

A lower limit on the number density of these $\mathrm{MeV}$ photons is

$$
n_{\gamma} \approx \frac{L_{f f}}{4 \pi c\left(2 r_{\mathrm{g}}\right)^{2} e_{\gamma}} \approx \frac{0.7 q_{f f} r_{\mathrm{g}}^{3}}{c r_{\mathrm{g}}^{2} e_{\gamma}} \sim \frac{10^{9} \dot{m}^{2}}{\alpha_{\mathrm{ss}}^{2} \eta^{2} M_{8}} \mathrm{~cm}^{-3},
$$

where $e_{\gamma}=3 \theta_{\mathrm{e}} m_{\mathrm{e}} c^{2}$. The production rate of $e^{ \pm}$pairs inside the magnetosphere due to $\gamma \gamma$-annihilation is approximately $\sigma_{\gamma \gamma} n_{\gamma}^{2} c(4 \pi / 3)\left(2 r_{\mathrm{g}}\right)^{3}$, where $\sigma_{\gamma \gamma} \approx \sigma_{\mathrm{T}} / 5$ is the cross-section of two-photon pair production. In steady state, this rate is balanced by the escape rate $\sim 4 \pi c\left(2 r_{\mathrm{g}}\right)^{2} n_{ \pm}$, allowing us to estimate

$$
n_{ \pm} \gtrsim 10^{6} \frac{\dot{m}^{4}}{\eta^{4} \alpha_{\mathrm{ss}}^{4} M_{8}} \mathrm{~cm}^{-3}
$$

The GJ density (ignoring its polar angle dependence) is determined by the accretion rate, $\dot{m}$, via Equations (8) and (18):

$$
n_{\mathrm{GJ}}=\frac{\Omega B}{2 \pi e c}=0.4\left(\frac{\beta_{m} \dot{m}}{\eta \alpha_{\mathrm{ss}} M_{\mathrm{bh}, 8}^{3}}\right)^{1 / 2} \mathrm{~cm}^{-3} .
$$

Thus, the multiplicity parameter is

$$
\kappa \gtrsim \frac{n_{ \pm}}{n_{\mathrm{GJ}}} \approx 6 \times 10^{6} \frac{\dot{m}^{7 / 2} M_{\mathrm{bh}, 8}^{1 / 2}}{\eta^{7 / 2} \alpha_{\mathrm{ss}}^{7 / 2} \beta_{m}^{1 / 2}} .
$$

The condition $\kappa<1$ places upper limit on the accretion rate as

$$
\dot{m} \lesssim 10^{-2} \frac{\eta \alpha_{\mathrm{ss}} \beta_{m}^{1 / 7}}{M_{8}^{1 / 7}} .
$$

Equation (66) allows an estimation of the characteristic inverse Compton cooling time of electrons in the photon field provided by the disk. For an electron with energy 


$$
\begin{aligned}
E=10^{6} m_{\mathrm{e}} c^{2} \gamma_{6} & \\
\lambda_{\mathrm{IC}}= & \frac{3}{4 \sigma_{\mathrm{t}}} \frac{m c^{2}}{n_{\gamma} e_{\gamma} 10^{6} \gamma_{6}} \sim \frac{\alpha_{\mathrm{ss}}^{2} \eta^{2} M_{8}}{\theta_{\mathrm{e}}} \frac{4 \times 10^{8} \mathrm{~cm}}{\gamma_{6} \dot{m}^{2}} \\
& \gtrsim \frac{0.2 r_{\mathrm{g}}}{\gamma_{6}} \frac{M_{8}^{2 / 7}}{\beta_{\mathrm{m}}^{2 / 7} \theta_{\mathrm{e}}} .
\end{aligned}
$$

\section{Appendix B Small Angle Limit}

It is believed that the emission from AGNs is mostly detected by observers located within the jet-beaming cone $\theta \leqslant \Gamma_{j}^{-1}$, but there are also some examples when the flares are detected from off-axis radio galaxies, e.g., M87 and IC 310. Sometimes it is convenient to measure the viewing angle in the jet-opening units:

$$
\theta=\alpha \Gamma_{\mathrm{j}}^{-1} \text {. }
$$

In particular, this parameter gives a simple relation between the jet Doppler factor and the Lorentz factor:

$$
\delta_{\mathrm{j}}=\frac{1}{\Gamma_{\mathrm{j}}\left(1-\beta_{\mathrm{j}} \cos \theta\right)}=\frac{2 \Gamma_{\mathrm{j}}}{1+\alpha^{2}},
$$

which is valid for $\alpha \ll \Gamma_{\mathrm{j}}$.

If the production region moves relativistically in the jet, then its Lorentz factor with respect to the observer is (see, e.g., Giannios et al. 2009)

$$
\Gamma_{\mathrm{em}}=\Gamma_{\mathrm{j}} \Gamma_{\mathrm{co}}\left(1+\beta_{\mathrm{j}} \beta_{\mathrm{co}} \cos \theta^{\prime}\right),
$$

where $\theta^{\prime}$ is the angle between the jet velocity and the outflow velocity. This angle is related to the angle in the observer frame via the aberration formula,

$$
\tan \theta=\frac{\beta_{\mathrm{co}} \sin \theta^{\prime}}{\Gamma_{\mathrm{j}}\left(\beta_{\mathrm{co}} \cos \theta^{\prime}+\beta_{\mathrm{j}}\right)} .
$$

The latter equation allows us to express the angle in the comoving reference frame

$$
\cos \theta^{\prime}=\frac{-\alpha^{2} \frac{\beta_{\mathrm{j}}}{\beta_{\mathrm{co}}} \pm \sqrt{\alpha^{4} \frac{\beta_{\mathrm{j}}^{2}}{\beta_{\mathrm{co}}^{2}}+\left(1-\alpha^{2} \frac{\beta_{\mathrm{j}}^{2}}{\beta_{\mathrm{co}}^{2}}\right)\left(1+\alpha^{2}\right)}}{1+\alpha^{2}} .
$$

One should account for the kinematic constraints that naturally appear in the above equation: $\left|\cos \theta^{\prime}\right| \leqslant 1$. Taking the solution with the + sign (which corresponds to a stronger enhancement in the region where two solutions are allowed) in the limit $\Gamma_{\mathrm{j}, \text { co }} \gg 1$, one obtains

$$
\beta_{\mathrm{co}} \cos \theta^{\prime} \simeq \frac{1-\alpha^{2}}{1+\alpha^{2}}-\frac{1}{2 \Gamma_{\mathrm{co}}^{2}},
$$

and consequently, the emitter Lorentz factor is

$$
\Gamma_{\mathrm{em}} \simeq \frac{2 \Gamma_{\mathrm{j}} \Gamma_{\mathrm{co}}}{1+\alpha^{2}}
$$

\section{References}

Abdo, A. A., Ackermann, M., Ajello, M., et al. 2011, ApJL, 733, L26 Abramowicz, M. A., Czerny, B., Lasota, J. P., \& Szuszkiewicz, E. 1988, ApJ, 332,646
Abramowski, A., Acero, F., Aharonian, F., et al. 2012, ApJ, 746, 151 Ackermann, M., Anantua, R., Asano, K., et al. 2016, ApJL, 824, L20

Aharonian, F., Akhperjanian, A. G., Bazer-Bachi, A. R., et al. 2007, ApJL, 664, L71

Albert, J., Aliu, E., Anderhub, H., et al. 2007, ApJ, 669, 862

Aleksić, J., Ansoldi, S., Antonelli, L. A., et al. 2014, Sci, 346, 1080

Araudo, A. T., Bosch-Ramon, V., \& Romero, G. E. 2010, A\&A, 522, A97

Araudo, A. T., Bosch-Ramon, V., \& Romero, G. E. 2013, MNRAS, 436, 3626

Barkov, M. V., Aharonian, F. A., Bogovalov, S. V., Kelner, S. R., \& Khangulyan, D. 2012a, ApJ, 749, 119

Barkov, M. V., Aharonian, F. A., \& Bosch-Ramon, V. 2010, ApJ, 724, 1517

Barkov, M. V., \& Baushev, A. N. 2011, NewA, 16, 46

Barkov, M. V., Bosch-Ramon, V., \& Aharonian, F. A. 2012b, ApJ, 755, 170

Barkov, M. V., \& Komissarov, S. S. 2016, MNRAS, 458, 1939

Barniol Duran, R., Tchekhovskoy, A., \& Giannios, D. 2016, arXiv:1612.06929

Bednarek, W., \& Protheroe, R. J. 1997, MNRAS, 287, L9

Begelman, M. C., Blandford, R. D., \& Rees, M. J. 1984, RvMP, 56, 255

Beskin, V. S. 2010, MHD Flows in Compact Astrophysical Objects (Berlin: Springer)

Beskin, V. S., Istomin, Y. N., \& Parev, V. I. 1992, SvA, 36, 642

Beskin, V. S., \& Nokhrina, E. E. 2006, MNRAS, 367, 375

Bisnovatyi-Kogan, G. S., \& Blinnikov, S. I. 1977, A\&A, 59, 111

Bisnovatyi-Kogan, G. S., \& Lovelace, R. V. E. 2007, ApJL, 667, L167

Blandford, R. D., \& Königl, A. 1979, ApJ, 232, 34

Blandford, R. D., \& Znajek, R. L. 1977, MNRAS, 179, 433

Bonnoli, G., Ghisellini, G., Foschini, L., Tavecchio, F., \& Ghirlanda, G. 2011, MNRAS, 410,368

Bosch-Ramon, V., Perucho, M., \& Barkov, M. V. 2012, A\&A, 539, A69

Broderick, A. E., \& Tchekhovskoy, A. 2015, ApJ, 809, 97

Celotti, A., Fabian, A. C., \& Rees, M. J. 1998, MNRAS, 293, 239

Cui, Y.-D., Yuan, Y.-F., Li, Y.-R., \& Wang, J.-M. 2012, ApJ, 746, 177

de la Cita, V. M., Bosch-Ramon, V., Paredes-Fortuny, X., Khangulyan, D., \& Perucho, M. 2016, A\&A, 591, A15

Falco, E. E., Kurtz, M. J., Geller, M. J., et al. 1999, PASP, 111, 438

Ferrarese, L., \& Merritt, D. 2000, ApJL, 539, L9

Gebhardt, K., \& Thomas, J. 2009, ApJ, 700, 1690

Gebhardt, K., Bender, R., Bower, G., et al. 2000, ApJL, 539, L13

Giannios, D., Uzdensky, D. A., \& Begelman, M. C. 2009, MNRAS, 395, L29

Giannios, D., Uzdensky, D. A., \& Begelman, M. C. 2010, MNRAS, 402, 1649

Goldreich, P., \& Julian, W. H. 1969, ApJ, 157, 869

Golkhou, V. Z., Butler, N. R., \& Littlejohns, O. M. 2015, ApJ, 811, 93

Gregori, G., Miniati, F., Ryu, D., \& Jones, T. W. 2000, ApJ, 543, 775

Hayashida, M., Nalewajko, K., Madejski, G. M., et al. 2015, ApJ, 807, 79

Hirotani, K., \& Pu, H.-Y. 2016, ApJ, 818, 50

Hirotani, K., Pu, H.-Y., Chun-Che Lin, L., et al. 2016, ApJ, 833, 142

Kadler, M., Eisenacher, D., Ros, E., et al. 2012, A\&A, 538, L1

Khangulyan, D. V., Barkov, M. V., Bosch-Ramon, V., Aharonian, F. A., \& Dorodnitsyn, A. V. 2013, ApJ, 774, 113

Komissarov, S. S. 1994, MNRAS, 266, 649

Komissarov, S. S. 2003, MNRAS, 341, 717

Komissarov, S. S. 2004, MNRAS, 350, 427

Komissarov, S. S., Barkov, M. V., Vlahakis, N., \& Königl, A. 2007, MNRAS, 380,51

Komissarov, S. S., Vlahakis, N., Königl, A., \& Barkov, M. V. 2009, MNRAS, 394, 1182

Levinson, A. 2000, PhRvL, 85, 912

Levinson, A., Melrose, D., Judge, A., \& Luo, Q. 2005, ApJ, 631, 456

Levinson, A., \& Rieger, F. 2011, ApJ, 730, 123

Li, Y.-R., Yuan, Y.-F., Wang, J.-M., Wang, J.-C., \& Zhang, S. 2009, ApJ, 699,513

Lovelace, R. V. E. 1976, Natur, 262, 649

Lynden-Bell, D. 1969, Natur, 223, 690

Lyubarsky, Y. E. 2005, MNRAS, 358, 113

McKinney, J. C., Tchekhovskoy, A., \& Blandford, R. D. 2012, MNRAS, 423, 3083

McKinney, J. C., \& Uzdensky, D. A. 2012, MNRAS, 419, 573

Nakamura, F., McKee, C. F., Klein, R. I., \& Fisher, R. T. 2006, ApJS, 164, 477

Narayan, R., \& Yi, I. 1994, ApJL, 428, L13

Narayan, R., \& Yi, I. 1995a, ApJ, 444, 231

Narayan, R., \& Yi, I. 1995b, ApJ, 452, 710

Neronov, A., \& Aharonian, F. A. 2007, ApJ, 671, 85

Petropoulou, M., Giannios, D., \& Sironi, L. 2016, MNRAS, 462, 3325

Pittard, J. M., Hartquist, T. W., \& Falle, S. A. E. G. 2010, MNRAS, 405, 821

Porth, O., \& Komissarov, S. S. 2015, MNRAS, 452, 1089 
Pozanenko, A., \& Loznikov, V. 2002, in Lighthouses of the Universe: The Most Luminous Celestial Objects and Their Use for Cosmology, ed. M. Gilfanov, R. Sunyeav, \& E. Churazov (Berlin: Springer), 194 Rieger, F. M. 2011, IJMPD, 20, 1547

Rieger, F. M., \& Aharonian, F. A. 2008, A\&A, 479, L5

Rieger, F. M., \& Volpe, F. 2010, A\&A, 520, A23

Romoli, C., Taylor, A. M., \& Aharonian, F. 2017, APh, 88, 38

Ruderman, M. A., \& Sutherland, P. G. 1975, ApJ, 196, 51

Ruffini, R., \& Wilson, J. R. 1975, PhRvD, 12, 2959

Salpeter, E. E. 1964, ApJ, 140, 796

Shakura, N. I., \& Sunyaev, R. A. 1973, A\&A, 24, 337
Sijbring, D., \& de Bruyn, A. G. 1998, A\&A, 331, 901

Sironi, L., Giannios, D., \& Petropoulou, M. 2016, MNRAS, 462, 48

Stern, B. E., \& Poutanen, J. 2006, MNRAS, 372, 1217

Striani, E., Vercellone, S., Tavani, M., et al. 2010, ApJ, 718, 455

Sturrock, P. A. 1971, ApJ, 164, 529

Timokhin, A. N. 2010, MNRAS, 408, 2092

Timokhin, A. N., \& Arons, J. 2013, MNRAS, 429, 20

Urry, C. M., \& Padovani, P. 1995, PASP, 107, 803

Vovk, I., \& Babić, A. 2015, A\&A, 578, A92

Wang, C.-C., \& Zhou, H.-Y. 2009, MNRAS, 395, 301

Zel'dovich, Y. B., \& Novikov, I. D. 1966, SvPhU, 8, 522 